\title{
Dark Matter Detection with the DEAP/CLEAN Detectors
}

\author{
Marek Kos ${ }^{* \dagger}$ \\ Syracuse University \\ E-mail: mskos@phy.syr.edu
}

The Dark matter Experiment using Argon Pulse shape discrimination (DEAP) and Cryogenic Low Energy Astrophysics with Noble liquids (CLEAN) detectors are single phase dark matter direct detection experiments. Single phase experiments have the advantage of being easily scalable to large detector volumes without significant loss of detection efficiency. The next phase of experiments, MiniCLEAN and DEAP3600, are currently under construction with expected sensitivities to the WIMP-nucleon cross section of $2 \times 10^{-45} \mathrm{~cm}^{2}$ for MiniCLEAN and $10^{-46} \mathrm{~cm}^{2}$ for DEAP3600

35th International Conference of High Energy Physics

July 22-28, 2010

Paris, France

*Speaker.

${ }^{\dagger}$ On behalf of the DEAP/CLEAN Collaboration 


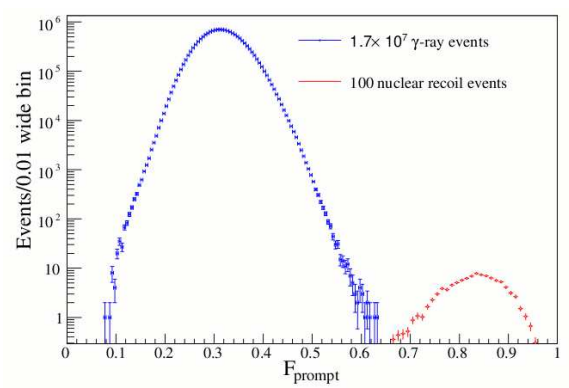

Figure 1: Fprompt for gamma and neutron calibration data. Figure taken from [1]

\section{Pulse Shape Discrimination in Noble Liquids}

The time dependence of scintillation light in noble liquids is different for heavy ionizers like alphas and recoiling nuclei compared to light ionizing particles (betas and gammas). The scintillation light is produced in the decay of either singlet or triplet excited states. The triplet state decays slower than the singlet state. The triplet state is suppressed for nuclear recoils, therefore scintillation light from nuclear recoils is more prompt than for electron recoils. The triplet and singlet excited states have very different decay times in liquid argon making argon a good choice as a target liquid.

\section{Background Rejection in DEAP-1}

Electron-recoil background rejection has been demonstrated in a small scale detector ( $7 \mathrm{~kg}$ of liquid argon) called DEAP-1. This detector was operated at Queen's University in an above ground lab. The fraction of prompt scintillation light to total scintillation light, Fprompt, was used to discriminate electron recoils from nuclear recoils. The prompt time window was set from $-50 \mathrm{~ns}$ before the start of the pulse to $150 \mathrm{~ns}$ after. Figure 1 shows the discrimination based on Fprompt between gamma and neutron calibration events. The discrimination against electron recoil type events based on Fprompt is expected to be about $10^{9}$ to 1 .

\section{The MiniCLEAN and DEAP3600 Detectors}

The next phase of larger detectors, MiniCLEAN and DEAP3600, are currently under construction. MiniCLEAN will have a total liquid argon mass of $500 \mathrm{~kg}$, with a $150 \mathrm{~kg}$ fiducial volume. It will be instrumented with 91 Photomultiplier Tubes (PMTs). The outer vacuum vessel has already been built, and the inner vessel is currently being manufactured. DEAP3600 will be a larger detector with a $1000 \mathrm{~kg}$ fiducial volume instrumented with 266 PMTs. Both detectors will be at the SNOLAB underground laboratory in Sudbury Ontario Canada. The expected start date for MiniCLEAN is late 2011 and for DEAP3600 it is late 2012.

\section{References}

[1] M. G. Boulay et al., arXiv:0904.2930 [astro-ph.IM]. 